\title{
LA SEMICONTINUTTÉ ET LA PROPRIÉTÉ DE BAIRE
}

\author{
Z. GRANDE AND S. STAWIKOWSKA
}

\begin{abstract}
Let $X, Y$ be two metric separable and complete spaces and $R$ be a set of all reals numbers. If all sections $f_{x}(y)=f(x, y)(x \in X$ and $y \in Y)$ of a function $f: X \times Y \rightarrow R$ are almost qualitative upper semiequicontinuous and upper semicontinuous [upper semiequicontinuous] and if all sections $f^{y}(x)=f(x, y)$ have the Baire property [are borelien], then the function $f$ has the Baire property [is borelien].
\end{abstract}

Soient $\left(X, \xi_{1}\right)$ et $\left(Y, \xi_{2}\right)$ deux espaces métriques, séparables et complets et $R$ l'espace des nombres réels. Étant donné une fonction $f: X \times Y \rightarrow R$ et $x_{0} \in X$ et $y_{0} \in Y$ étant fixés, les fonctions d'une variable $f_{x_{0}}(y)=f\left(x_{0}, y\right)$ et $f^{y_{0}}(x)=f\left(x, y_{0}\right)(x \in X$ et $y \in Y)$ sont dites coupes de la fonction $f$ relativement à $x_{0}$ et $y_{0}$ respectivement.

On said, par exemple, que si toutes les coupes $f_{x}$ de la fonction $f$ sont continues et toutes ses coupes $f^{y}$ ont la propriété de Baire, la fonction $f$ a la propriété de Baire.

D'autre part il resulte des théorèmes 15.5 et $15.4 \mathrm{du}$ livre [2] l'existence d'une fonction $f: R^{2} \rightarrow R$ qui n'a pas de la propriété de Baire et dont toutes les coupes $f_{x}$ et $f^{y}$ sont semicontinues supérieurement et discontinues au plus en deux points.

Dans cet article cous introduisons certaines propriétés des coupes $f_{x}$ et $f^{y}$ d'une fonction $f: X \times Y \rightarrow R$ qui impliquent la propriété de Baire de la fonction $f$.

DÉFINITION 1. On dit qu'une fonction $g: X \rightarrow R$ est qualitativement semicontinue supérieurement [presque qualitativement semicontinue supérieurement] au point $x_{0} \in X$ lorsqu'il existe un ensemble $A \subset X$ ayant la propriété de Baire et résiduel dans certain entourage du point $x_{0}$ [ayant la propriété de Baire et de deuxième catégorie dans chaque entourage du point $x_{0}$ ] tel que $x_{0} \in A$ et la fonction partielle $g \mid A$ est semicontinue supérieurement au point $x_{0}$.

DÉFInITION 2. On dit que les fonctions $g_{t}: X \rightarrow R$, ou $t \in T$ et $T$ est un ensemble d'indices, sont qualitativement semiéquicontinues supérieurement [presque qualitativement semiéquicontinues supérieurement] au point $x_{0}$ lors qu' il existe un ensemble $A \subset X$ ayant la propriété de Baire et résiduel dans certain entourage du point $x_{0}$ [ayant la propriété de Baire et de deuxième catégorie dans chaque entourage du point $x_{0}$ ] tel que $x_{0} \in A$ et les fonctions partielles $g_{t} \mid A, t \in T$, sont semiéquicontinues supérieurement au point $x_{0}$

Received by the editors June 15, 1977.

AMS (MOS) subject classifications (1970). Primary 26A35.

(c) 1979 American Mathematical Society 0002-9939/79/0000-0461/\$02.25 
(c'est-à-dire:

$$
\left.\bigwedge_{\varepsilon>0} \bigvee_{\delta>0} \bigwedge_{x \in A} \bigwedge_{t \in T}\left[\xi_{1}\left(x, x_{0}\right)<\delta \Rightarrow g_{t}(x)-g_{t}\left(x_{0}\right)<\varepsilon\right]\right) \text {. }
$$

ThÉRÈmE 1. Si toutes les coupes $f_{x}$ d'une fonction $f: X \times Y \rightarrow R$ sont qualitativement semiéquicontinues supérieurement [presque qualitativement semiéquicontinues supérieurement] au point $y_{0}$ et si toutes les coupes $f^{y}$ de cette fonction sont qualitativement semicontinues supérieurement [presque qualitativement semicontinues supérieurement] au point $x_{0}$, la fonction $f$ est qualitativement semicontinues supérieurement [presque qualitativement semicontinue supérieurement] au point $\left(x_{0}, y_{0}\right)$.

DÉmonstration. La coupe $f^{y_{0}}$ étant qualitativement semicontinue supérieurement au point $x_{0}$, il existe un ensemble $A \subset X$, ayant la propriété de Baire, contenant le point $x_{0}$ et residuel dans certain entourage $U$ du point $x_{0}$ tel que la fonction partielle $f^{y_{0}} \mid A$ est semicontinue supérieurement au point $x_{0}$. Les coupes $f_{x}$ étant qualitativement semiéquicontinues supérieurement au point $y_{0}$, il existe un ensemble $B \subset Y$ ayant la propriété de Baire, contenant $y_{0}$ et résiduel dans certain entourage $V$ du point $y_{0}$ tel que les fonctions partielles $f_{x} \mid B$ sont semiéquicontinues supérieurement au point $y_{0}$. Remarquons que l'ensemble $C=A \times B$ a la propriété de Baire, contient le point $\left(x_{0}, y_{0}\right)$ et est résiduel dans l'entourage $U \times V$ du point $\left(x_{0}, y_{0}\right)$.

Démontrons encore que la fonction partielle $f \mid C$ est semicontinue supérieurement au point $\left(x_{0}, y_{0}\right)$. Fixons le nombre $\varepsilon>0$. Il existe un nombre $\delta>0$ tel que

$$
f\left(x, y_{0}\right)-f\left(x_{0}, y_{0}\right)<\varepsilon / 2
$$

pour tout $x \in A \cap\left\{t \in X: \xi_{1}\left(t, x_{0}\right)<\delta_{1}\right\}$ et il existe un nombre $\delta_{2}>0$ tel que

$$
f(x, y)-f\left(x, y_{0}\right)<\varepsilon / 2
$$

pour tout $y \in B \cap\left\{t \in Y: \xi_{2}\left(t, y_{0}\right)<\delta_{2}\right\}$ et pour tout $x \in X$. Posons $\delta=$ $\min \left(\delta_{1}, \delta_{2}\right)$ et remarquons que

$$
\begin{aligned}
f(x, y)-f\left(x_{0}, y_{0}\right) & =f(x, y)-f\left(x, y_{0}\right)+f\left(x, y_{0}\right)-f\left(x_{0}, y_{0}\right) \\
& <\varepsilon / 2+\varepsilon / 2=\varepsilon
\end{aligned}
$$

pour tout $(x, y) \in C \cap\left\{(u, v) \in X \times Y:\left(\xi_{1} \times \xi_{2}\right)\left[(u, v), \quad\left(x_{0}, y_{0}\right)\right]<\delta\right\}$, d'où il vient que la fonction $f \mid C$ est semicontinue supérieurement au point $\left(x_{0}, y_{0}\right)$.

La démonstration du fait que la fonction $f$ est presque qualitativement semicontinue supérieurement au point $\left(x_{0}, y_{0}\right)$ est analogue.

ThÉorème 2' ([1, Théorème 1]). Pour qu'une fonction $f: X \rightarrow R$ ait la propriété de Baire, il faut et il suffit qu'elle satisfasse à la condition

(I) il existe, pour tout nombre $\varepsilon>0$ et pour tout ensemble $A \subset X$ ayant la propriété de Baire et de deuxième catégorie, un ensemble $B \subset A$ ayant la propriété de Baire et de deuxième catégorie tel que $\csc _{B} f \leqslant \varepsilon$. 
ThÉORÈme 2. Si toutes les coupes $f_{x} d$ 'une fonction $f: X \times Y \rightarrow R$ sont presque qualitativement semiéquicontinues supérieurement et semicontinues supérieurement en tout point $y \in Y$ et si toutes ses coupes $f^{y}$ ont la propriété de Baire, la fonction $f^{x}$ la propriété de Baire.

DÉmonstration. Sans restreindre la généralité on peut supposer que la fonction $f$ est bornée. Supposons au contraire, que la fonction $f$ n'ait pas la propriété de Baire. Il existe donc, d'après le Théorème $2^{\prime}$, un nombre $\varepsilon>0$ et un ensemble $A \subset X \times Y$ de deuxième catégorie et ayant la propriété de Baire tels que, quel que soit l'ensemble $B \subset A$ ayant la propriété de Baire et de duexième catégorie, $\csc _{B} f>\varepsilon$. Désignons par a le nombre

$\sup \left\{\inf _{A-Z} f(x): Z \subset X\right.$ et $Z$ est un ensemble de première catégorie $\}$.

Posons $A_{1}=\{(x, y) \in A: a \leqslant f(x, y)\}$. Remarquons que l'ensemble $A-$ $A_{1}$ est de première catégorie. Désignons par $C$ l'ensemble

$$
\left\{(x, y) \in A_{1}: f(x, y)<a+\varepsilon / 4\right\} \text {. }
$$

L'ensemble $C$ est de deuxième catégorie.

Soit $\left\{S_{n}\right\}$ la suite des ensembles ouverts d'une base dénombrable de l'espace $Y$. Les coupes $f_{x}$ étant semicontinues supèrieurement, il existe pour tout point $(x, y) \in C$ un ensemble ouvert $S(x, y)$ de la suite $\left\{S_{n}\right\}$ tel que

$$
f(x, v)-f(x, y)<\varepsilon / 4 \text { pour tout } v \in S(x, y)
$$

Soit $S_{n_{0}}$ l'ensemble ouvert de la suite $\left\{S_{n}\right\}$ tel que l'ensemble

$$
D=\left\{(x, y) \in C: \text { au point }(x, y) \text { correspond l'ensemble } S(x, y)=S_{n_{0}}\right\}
$$

est de deuxième catégorie.

Les coupes $f_{x}$ étant presque qualitativement semiéquicontinues supérieurement, il existe un point $\left(x_{0}, y_{0}\right) \in D$ et un ensemble $K \subset S_{n_{0}}$ ayant la propriété de Baire, contenant le point $y_{0}$, de deuxième catégorie tel que

$$
f(x, y)-f\left(x, y_{0}\right)<\varepsilon / 4,
$$

quels que soient le point $x \in X$ et le point $y \in K$, pour lesquels l'ensemble $(X \times K) \cap D=F$ est de deuxième catégorie. Remarquons que l'ensemble

$$
E=\left\{x \times X: f\left(x, y_{0}\right)<a+\varepsilon / 2\right\} \times K
$$

a la propriété de Baire. L'ensemble $A_{1} \cap E$ a également la propriété de Baire et il est de deuxième catégorie, comme il contient un sous-ensemble de deuxième catégorie $(F)$.

Reste àvoir que $\operatorname{osc}_{A_{1} \cap E} f \leqslant \varepsilon$. En effet, si $\left(x_{1}, y_{1}\right) \in A_{1} \cap E, f\left(x_{1}, y_{1}\right) \geqslant a$. D'autre part, $\left(x_{1}, y_{1}\right) \in E$, on a donc que $f\left(x_{1}, y_{0}\right)<a+\varepsilon / 2$. Par conséquent, on a

$$
f\left(x_{1}, y_{1}\right)<f\left(x_{1}, y_{0}\right)+\varepsilon / 4<a+\varepsilon / 2+\varepsilon / 4<a+\varepsilon,
$$

d'où notre théorème. 
Problème 1. Une fonction $f: X \times Y \rightarrow R$ dont les coupes $f_{x}$ sont presque qualitativement semiéquicontinues supérieurement en tout point $y \in Y$ et dont les coupes $f^{y}$ ont la propriété de Baire doit-elle avoir la propriété de Baire?

THÉORÈmE 3. Si toutes les coupes $f_{x}$ d'und fonction $f: X \times Y \rightarrow R$ sont semiéquicontinues et si toutes ses coupes $f^{y}$ sont boreliennes, la fonction $f$ est borelienne.

Démonstration. Fixons le nombre $a$. Nous démontrerons que l'ensemble $\{(x, y) \in X \times Y: f(x, y)<a\}$ est borelien. Fixons les nombres naturels $n$ et $k$. Les coupes $f_{x}$ étant semiéquicontinues supérieurement, il existe une suite $\left\{y_{n k l}\right\}$ de points de l'espace $Y$ et une suite $\left\{U_{n k l}\right\}$ d'ensembles ouverts de l'espace $Y$ tels que $y_{n k l} \in U_{n k l}$ pour $l=1,2, \ldots, Y \subset \cup_{L=1}^{\infty} U_{n k l}$, le diamètre $d\left(U_{n k l}\right)$ de l'ensemble $U_{n k l}$ est $<1 / k$ pour $l=1,2, \ldots$ et $f(x, v)$ $f\left(x, y_{n k l}\right)<4^{-n}$ pour tout $x \in X$ et pour tout $v \in U_{n k l}$.

On a les inégalités

$$
\begin{aligned}
&\{(x, y) \in X \times Y: f(x, y)<a\} \\
& \\
& \supset \bigcup_{k, l}\left[\left\{x \in X: f\left(x, y_{n k l}\right)<a-1 / n\right\} \times U_{n k l}\right] \\
& \supset\{(x, y) \in X \times Y: f(x, y)<a-1 / n\},
\end{aligned}
$$

quel que soit le nombre naturel $n$. En effet, si $\left(x_{0}, y_{0}\right) \in\left\{x \in X: f\left(x, y_{n k l}\right)<\right.$ $a-1 / n\} \times U_{n k l}$, on a $f\left(x_{0}, y\right)<f\left(x_{0}, y_{n k l}\right)+4^{-n}<a-1 / n+4^{-n}<a$. D'autre part, si $f\left(x_{0}, y_{0}\right)<a-1 / n$, il existe deux nombres naturels $l, k$ tels que $y_{0} \in U_{n k l}$ et $f\left(x_{0}, v\right)<a-1 / n$ pour tout $v \in U_{n k l}$. Par conséquent $f\left(x_{0}, y_{n k l}\right)<a-1 / n$ et $\left(x_{0}, y_{0}\right) \in\left\{x \in X: f\left(x, y_{n k l}\right)<a-1 / n\right\} \times U_{n k l}$.

L'ensemble

$$
\left\{x \in X: f\left(x, y_{n k l}\right)<a-1 / n\right\} \times U_{n k l}
$$

étant borelien, quels que soient les nombres naturels $n, k, l$, l'ensemble

$$
\begin{aligned}
\{(x, y) \in X & \times Y: f(x, y)<a\} \\
& =\bigcup_{n, k, l}\left[\left\{x \in X: f\left(x, y_{n k l}\right)<a-1 / n\right\} \times U_{n k l}\right]
\end{aligned}
$$

est le même, d'où notre théorème.

De la démonstration du Théorème 3 résulte la remarque suivante:

REMARQUE 1. Si toutes les coupes $f_{x} d$ 'une fonction $f: X \times Y \rightarrow R$ sont semiéquicontinues supérieurement et si toutes ses coupes $f^{y}$ sont de classe de Baire $\alpha$, la fonction $f$ est de classe de Baire $\alpha+1$.

Problème 2. Existe-t-il une fonction $f: X \times Y \rightarrow R$ qui n'est pas de première classe de Baire et dont les coupes $f_{x}$ sont semiéquicontinues supérieurement et dont les coupes $f^{y}$ sont de première classe de Baire. 


\section{Travaux Cités}

1. Z. Grande, La propriété de Baire des fonctions de deux variables ponctuelement discontinues par rapport à une variable, Bull. Acad. Polon. Sci. Sér. Sci. Math. Astronom. Phys. 25 (1977), 535-539.

2. J. Oxtoby, La mesure et la catégorie, Moscou, 1974. (Russe)

Ul. Malborsta 84/87, 82-300 Elblag, Poland 